\title{
Comparative Study for Prediction Accuracy of RANS Turbulence Models: Multi-Phase Flow in the Cavitating Venturi
}

\author{
Gong-Hee Lee ${ }^{1}$ \\ ${ }^{1}$ Department of Regulatory Assessment, Korea Institute of Nuclear Safety \\ 62 Gwahak-ro, Daejeon, 34142 Republic of Korea \\ ghlee@kins.re.kr
}

\section{Extended Abstract}

Nuclear power plant operators have conducted in-service testing (IST) to verify the safety functions of safety-related pumps and valves, and to monitor the degree of vulnerability over time during reactor operation [1]. The auxiliary feedwater system is one of the representative IST-related system and a cavitating venturi is installed to prevent cavitation due to pump runout and minimize other adverse effects resulting from the supply of excessive auxiliary feedwater flows [2]. A cavitating venturi generally consists of an upstream, contraction, throat, diffusion and downstream section. Rapid flow acceleration and accompanying pressure drop may cause cavitation near the straight throat and diffusion section of venturi, which may result in degradation and structural damage [1].

Since cavitating flows involve complex turbulent two-phase flows, the accurate simulation of this flow by using the available computational fluid dynamics (CFD) software is still a great challenge [3]. Though the competitiveness of CFD is growing steadily due to rapid developments in computer hardware technology, computing resource is still a limiting factor for CFD simulations to produce completely accurate results for the prediction of the cavitation flow [3]. Thus, turbulence models are required to bridge the gap between the real flow and the statistically averaged equations [3].

Licensing applications supported by using CFD software are recently increasing for IST-related problems, but there is no domestic regulatory guideline for the comprehensive evaluation of CFD software. Therefore, from the nuclear regulatory perspective, it is necessary to perform the systematic assessment and prepare the domestic regulatory guideline for checking whether valid CFD software and the numerical modeling is used for IST-related problems (including cavitation flow) [3]. To achieve the above-mentioned goals, the prediction accuracy of different Reynolds-averaged Navier-Stokes (RANS)-based turbulence models for the analysis of cavitating flow inside a cavitating venturi was assessed in this study. Four different turbulence models available in the CFX R19.1, for example, standard k- $\varepsilon$ model, ReNomarlization Group (RNG) k- $\varepsilon$ model, Shear Stress Transport (SST) k- $\omega$ model, and Baseline Reynolds stress model, were chosen and the predicted results were compared with the measured data [4]. The flow inside the cavitating venturi was assumed to be steady, incompressible, turbulent and multi-phase flow. Unstructured hexahedral grid system, generated by a grid generation software, ICEM-CFD, was used. Detailed information for the analysis model, the numerical model, grid distribution, boundary conditions, and convergence criteria can be found in the author's previous study [1].

Major conclusions can be summarized as follows: (1) At the specified corresponding inlet velocity, the predicted static pressure drop with RNG k- $\varepsilon$ model showed the best agreement with the measured one. On the other hand, standard k- $\varepsilon$ model somewhat over-predicted the static pressure drop at inlet pressure above $160 \mathrm{kPa}$. (2) Baseline Reynolds stress models did not guarantee the improved prediction results compared to the 2-equation model (i.e. SST k- $\omega$ model) even though they solved the complex transport equations for the individual components of the Reynolds stress tensor.

\section{Acknowledgement}

This work was supported by the Nuclear Safety Research Program through the Korea Foundation Of Nuclear Safety (KOFONS) using the financial resource granted by the Nuclear Safety and Security Commission (NSSC) of the Republic of Korea (No. 1805007). This work was also supported by the Supercomputing Center/Korea Institute of Science and Technology Information with supercomputing resources including technical support (project number: KSC-2019-CRE0236). 


\section{References}

[1] G. H. Lee and J. H. Bae, "CFD simulation of cavitation flow inside a cavitating venturi using ANSYS CFX," in Transactions of the Korean Nuclear Society Spring Meeting, Jeju, Korea, 2020.

[2] Korea Hydro \& Nuclear Power, Final Safety Analysis Report - Shinkori 3 \& 4, 10.4.9. Auxiliary Feedwater System, 2019.

[3] G. H. Lee and J. H. Bae, "Prediction of cavitating flow inside a sharp-edged orifice using different RANS turbulence models," in 15 th Asian International Conferences on Fluid Machinery, Busan, Korea, AICFM15-091, 2019.

[4] H. Shi, M. Li, P. Nikrityuk and Q. Liu, "Experimental and numerical study of cavitation flows in venturi tubes: from CFD to an empirical model," Chem. Eng. Sci., vol. 207, pp. 672-687, 2019. 